\title{
Nephroprotective Effect of Low Viscosity Sodium Alginate on Lead Acetate-Induced Nephrotoxicity in Rats
}

\author{
Gagandeep Kaur ${ }^{1}$, Saurabh Sharma ${ }^{2 \star}$ \\ ${ }^{1}$ M.Pharm [Pharmacology], I.S.F College of Pharmacy, Moga, Punjab, INDIA. \\ ${ }^{2}$ M.Pharm,Phd, Professor and Head, School of Pharmaceutical Sciences, CT University, Ludhiana, INDIA.
}

\begin{abstract}
Objective: Renal failure in majority of population is considered to be idiopathic. Heavy metals like lead in drinking water are usually the hidden cause of these renal consequences. Thus, this study has been designed to investigate the effect of low viscosity sodium alginate (LvSA) in Lead-induced nephrotoxicity. Methods: Lead $10.2 \%$ lead acetate in drinking water for 5 weeks) was administered in rats to produce nephrotoxicity assessed in terms of histopathological changes, increase in serum creatinine, blood urea nitrogen, urinary $\mathrm{N}$-acetyl- $\beta$ D glucosaminidase (NAG), proteinuria, Thiobarbituric acid reactive substances (TBARS) and decrease in reduced glutathione level. Results : Lead acetate (LA) intoxication shown elevations in serum creatinine, Proteinuria, glomerular filtration rate (GFR), blood urea nitrogen (BUN), Thiobarbituric acid reactive substances (TBARS), altered urinary $\mathrm{N}$-acetyl- $\beta$ D glucosaminidase (NAG), kidney weight/ body weight ratio (KW/BW \%), and creatinine clearance that indicate renal damage. Treatment with Low viscosity sodium alginate (LvSA) show significant decrease in serum creatinine, BUN, urinary NAG, protein in urine, KW/BW \%, TBARS level and increase in GSH, creatinine clearance and GFR. Conclusion: Thus, on the basis of results it may be concluded that LvSA significantly attenuated Lead-induced nephrotoxicity.
\end{abstract}

Key words: Lead acetate nephrotoxicity, Glomerular blood flow, Oxidative stress, Low viscosity sodium alginate, Proximal convoluted tubules.

\section{INTRODUCTION}

Lead $(\mathrm{Pb})$ is a chemical element in the carbon group with atomic number 82 . It is a very widespread environmental pollutant and its industrial use has been declined due to its known devastating toxic effects. ${ }^{1}$ However, it remains in biological cycle, because of deterioration of lead paints used in the past, and in vehicle exhaust into soil and dust. The danger of lead toxicity has been known since ancient times, the clinical manifestations of which are termed plumbism. ${ }^{2}$ Depending upon the exposure and accumulation of lead it causes acute or chronic poisoning.

Among the toxicity profile of lead on various organs, kidney is especially susceptible to the damaging effects of lead due to its major role in the excretion of lead from the body and is a major cause of mortality. ${ }^{3}$ Lead damages cellular material and produces oxidative stress by increased production of free radicals and decreased availability of antioxidant reserves. ${ }^{4}$ It also interrupts enzyme activation and competitively inhibits trace mineral absorption. Lead poisoning may also cause reduction in renal excretion of 6-Quito-prostaglandin factor 1-alpha (a vasodilator), enhanced excretion of thromboxane (a vasoconstrictor) which make the kidney more vulnerable or prone to inflammation and favours the nephrotoxicity. Lead alters calcium homeostasis and binds to sulfhydryl proteins by interrupting structural protein synthesis. The kidneys play a major role in the excretion of lead from the body. ${ }^{5}$ and higher content of lead has been estimated in renal tissue. In addition,
Submission Date: 09-09-2017; Revision Date: 25-09-2017; Accepted Date: 27-10-2017

DOI: 10.5530/ijper.51.4s.83 Correspondence:

Dr. Saurabh Sharma, MPharm,Phd, Professor and Head, School of Pharmaceutical Sciences, CT University, Ludhiana, INDIA.

Phone no: 7307355443 E-mail:ssm.research@ gmail.com

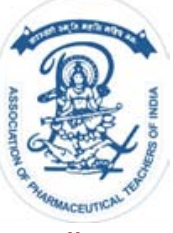

www.ijper.org 
lead causes mitochondrial damage, intracellular depletion of glutathione and apoptosis. ${ }^{6}$

Chelation that is multiple coordination bonds between ligand (also called chelating agents, or sequestering agents) and a single central atom. Chelation is used as a therapy to remove toxic metals from the body, contrast agents in MRI scanning and also applicable as nutritional supplements. Chelating agents vary in their properties like absorption from the digestive tract, penetration from various body tissues, and efficacy with individual heavy metal forgoing its removal and excretion. ${ }^{7}$ some chelates and metal complexes cross the blood-brain barrier more effectively than others. EDTA, DMSA and DMPS are commonly used synthetic chelating agents. On the other hand, natural polymers such as algal polysaccharides alginate. ${ }^{8}$ and chlorella. ${ }^{9}$ have also been gaining attention as potential adsorbents of heavy metals. Some previous case studies reported that modified citrus pectin plus alginate products have been used successfully to reduce lead. ${ }^{8} \mathrm{LvSA}$ has the mild chelating effect of heavy metals as a result is safe in therapeutic dosage. Sodium alginate, is a polysaccharide widely distributed in the cell walls of brown algae, where through quickly absorbing water makes viscous gum. Previous studies documented that LvSA treatment significantly reduce the urinary protein excretion and serum creatinine as well as vanquish the progression of glomerular lesions in immune complex glomerulonephritis. ${ }^{11}$ Moreover, the protective and reparative effects of sodium alginate on radiation stomatitis and suppression of radioactive absorption by this compound in animals and human subjects were investigated. In addition, it is also documented to attenuate hypertension, associated kidney damage. ${ }^{10}$ Therefore, we hypothesize that administration of LvSA may preserve renal function, prevent histological alterations in LA- induced nephrotoxicity with mild chelation and by suppressing oxidative stress. Additionally, LvSA will be evaluated with suitable biochemical and histopathological parameters for its possible Reno protective effect.

\section{MATERIAL AND METHODS}

The experimental protocol used in the present study was approved by the Institutional Animal Ethical Committee of ISF College of Pharmacy, Moga. Thirty rats of either sex were divided into 5 groups with 6 rats each. This study was carried out on wistar rats weighing approximately 180-250 g were used in this experimental study. Approved by experimental protocol by Institutional animal ethical committee (IAEC) (Date: December, 2013; Meeting No 8/2014/ Protocol) and Rats were provided by Guru Angad Dev Veterinary and Animal Sciences University (GADVASU), Ludhiana, Punjab. The rats housed in plastic cages in a temperature-controlled room were exposed to normal day and light cycle and were fed on standard chow diet and water ad libitum.

\section{Animal grouping as follows}

GROUP I: (Normal Control): Rats were maintained on standard food and water and no treatment was given. GROUP II (LA control): LA $0.2 \%$ in drinking water for 5 weeks.

GROUP III: LA ( $0.2 \%$ in drinking water) for 5 weeks +LvSA (25 mg/kg., i.p) for last 7 days.

GROUP IV: LA ( $0.2 \%$ in drinking water) for 5 weeks +LvSA (50 mg/kg., i.p) for last 7 days.

GROUP VII: LA ( $0.2 \%$ in drinking water) for 5 weeks +EDTA (1mg/kg., p.o) for last 7 days.

\section{Sample collection and biochemical assays}

Lead-induced nephrotoxicity was assessed in terms of decrease in urine volume, blood urea nitrogen (BUN), serum creatinine, proteinuria, glomerular filtration rate (GFR), N- acetyl- $\beta-D$ glucosaminase (NAG) activity in urine, creatinine clearance, oxidative stress (TBARS and GSH) parameters and histopathological changes in the kidney. Urine samples were collected with the help of metabolic cages (rats were individually kept in cages) after $24 \mathrm{~h}$ from the last dose for urinary protein and urinary creatinine determination in cylinder containing sodium azide $0.1 \%$ to minimize bacterial growth. Blood samples were collected for determination of serum creatinine and blood urea nitrogen (BUN) by retro-orbital sinus.

\section{Estimation of serum and renal parameters}

The urine creatinine and serum creatinine concentration were estimated by previously described alkaline picrate method. ${ }^{12}$ using commercially available kits (Coral clinical system, Goa, India). Proteinuria was estimated by pyrogallol red method. ${ }^{13}$ using the commercially available kit (Coral clinical system, Goa, India). BUN was estimated by Berthelot method. ${ }^{14}$ using the commercially available kit (Coral clinical system, Goa, India). The glomerular filtration rate was calculated using the method described by. ${ }^{15}$ who demonstrated that in rats GFR can be evaluated rather accurately based on serum creatinine concentration and body mass. It is argued that GFR is proportional to the ratio of body mass and serum creatinine with a correlation coefficient $r^{2}=0.94$. Estimation of urinary NAG was done by following method: 


\section{Estimation of NAG activity in urine}

The principle of the assay is that the NAG catalyzes the hydrolysis of $\mathrm{p}$-nitrophenyl $\mathrm{N}$ - acetyl- $\beta$-D- glucosaminide to $\mathrm{p}$ - nitrophenol and $\mathrm{N}$ - acetyl glucosamine. The liberated $\mathrm{p}$ - nitrophenol is proportional to the enzymatic activity.

$0.5 \mathrm{ml}$ of fresh urine sample and $0.5 \mathrm{ml}$ of distilled water the final $\mathrm{pH}$ being 4.4 was incubated for $1 \mathrm{~h}$ at $38^{\circ} \mathrm{C}$. Then, $4 \mathrm{ml}$ of glycine $-\mathrm{NaOH}$ buffer $\mathrm{pH} 10.5$ was added and the solution was centrifuged. The liberated p- nitro phenol in the supernatant was measured spectrophotometrically $430 \mathrm{~nm}$. NAG activity for a specimen of urine is expressed in $\mathrm{U} / \mathrm{g}$ of creatinine. The standard curve of $\mathrm{p}$ - Nitro phenol for estimation of activity of $\mathrm{N}$ - acetyl- $\beta$-D-glucosaminase was plotted. ${ }^{14}$

\section{Kidney weight/ Body weight ratio}

It was determined as an index of glomerular, mesengial expansion and renal fibrosis. Rats were sacrificed by cervical dislocation; kidney was excised by opening the abdominal cavity. Kidney weight/body weight (KW/ BW) $\%$ was calculated according to an earlier described formula. ${ }^{16,17}$

\section{Oxidative stress}

Renal oxidative stress was assessed in term of renal thiobarbituric acid reactive substance (TBARS) and reduced glutathione (GSH). The kidney was dissected out and washed in ice cold isotonic saline and weighed. The kidney was then minced, and a homogenate $(10 \% \mathrm{w} / \mathrm{v})$ was prepared in chilled phosphate buffer solution. Centrifuged at 10,000 $\mathrm{g}$ for $20 \mathrm{~min}$ and the supernatant was used for estimating TBARS and GSH. Renal TBARS was estimated according to the method described earlier. ${ }^{18}$ GSH level was estimated by the method as described earlier. ${ }^{19}$

\section{Histopathological study}

One kidney from each rat in each group was fixed in 10\% formalin solution. Changes in glomeruli were assessed morphologically and histologically as described by. ${ }^{20}$ in $3 \mu \mathrm{m}-5 \mu \mathrm{m}$ thickness sections and stained with hematoxylin and eosin to assess the pathological changes that occurred in the glomeruli using light microscope.

\section{Statistical Analysis}

All values were expressed as mean \pm SD. Various biochemical parameters, i.e. Blood urea nitrogen, serum creatinine, urinary proteins, GFR, creatinine clearance, NAG levels were statistically analyzed using one-way ANOVA followed by Tukey's multiple comparison test.
The $\mathrm{p}$ value of less than 0.05 was considered to be statistically significant.

\section{RESULTS}

\section{Effect of LvSA on serum and renal parameters}

The serum and renal parameters were noted to be increased markedly in LA $(0.2 \%$ in drinking water for 5 days) induced nephrotoxicity rats. Treatment with LvSA (25 and $50 \mathrm{mg} / \mathrm{kg}$ i.p., for last 7 days). ${ }^{11}$ significantly reduced the LA induced increase in serum creatinine, BUN, urinary NAG and proteinuria, while creatinine clearance and GFR levels were significantly increased. Moreover, treatment with EDTA $(1 \mathrm{mg} / \mathrm{kg}$ p.o., last 7 days) also showed protective effect in LA induced nephrotoxicity rats Figure 1-6.

\section{Effect of LvSA on KW/BW\%}

LA administered rats showed significant $(\mathrm{P}<0.05)$ increased $\mathrm{KW} / \mathrm{BW} \%$. Treatment with $\mathrm{LvSA}$ significantly reduced $(\mathrm{p}<0.05) \mathrm{KW} / \mathrm{BW} \%$ in nephrotoxic rats (Figure 7).

\section{Effect of LvSA on \% increase in body weight and urine output}

No significant change was seen in \% increase in body weight and $\%$ increase in urine output Table 1.

\section{Effect of LvSA on oxidative stress}

Administration of LA significantly increased ( $p<0.05)$ TBARS level and decreased $(p<0.05)$ GSH level in the kidney; both TBARS and GSH are index of increased oxidative stress. Treatment with LvSA in LA administered rats showed significantly decreased $(\mathrm{p}<0.05)$ oxidative stress (Figure 8).

\section{Effect of LvSA on histology of kidney}

Microscopic examination of renal tissues stained with $\mathrm{H}$ and $\mathrm{E}$ revealed that there were normal renal glomeruli surrounded by a capsule and normal proximal, distal and convoluted tubules in normal control group. However, degeneration, desquamation, intracellular edema, glomerulus narrowing and necrosis were observed in epithelial cells of the proximal tubules in rats of LA control group. Degenerated and desquamated epithelial cells were in lumens of tubules. The large number of inflammatory cells infiltrated in the form of mononuclear cells and intertubular hemorrhage in the renal sections of this group. Glomerulus congestion and swelling were also observed, whereas there were mild lesions in the glomerulus and tubules of the renal tissues of rats treated with LA + LvSA when compared with the LA alone administered. Moreover, treatment with EDTA 


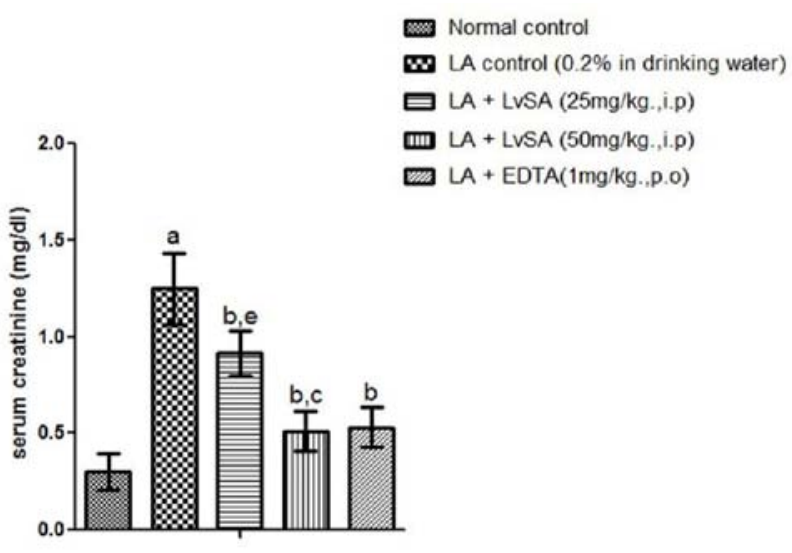

Figure 1: : Effect of low viscosity sodium alginate (SA) on serum creatinine $(\mathrm{mg} / \mathrm{dl})$. All values are represented as mean \pm SD. ${ }^{a} P<0.05$ vs normal control; ${ }^{b} P<0.05$ vs $L A$ control $(0.2 \%$ in drinking water); ${ }^{c} \mathrm{P}<0.05$ vs LvSA ( $25 \mathrm{mg} / \mathrm{kg}$., i.p).

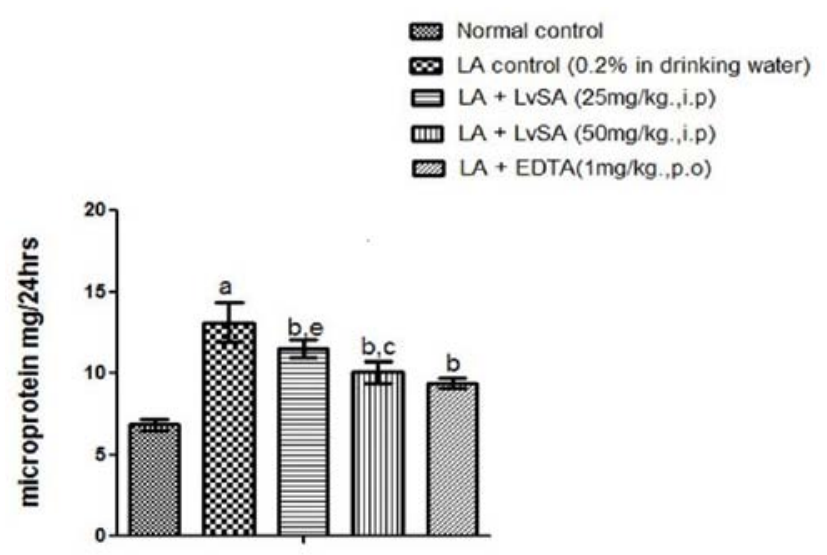

Figure 4: Effect of low viscosity sodium alginate (LvSA) on urinary protein $(\mathrm{mg} / 24 \mathrm{hr})$. All the values are represented in mean $\pm \mathrm{SD}$. ${ }^{\mathrm{P}} \mathrm{P}<0.05$ vs normal control; ${ }^{\mathrm{b}} \mathrm{P}<0.05$ vs $\mathrm{LA}$ control ( $0.2 \%$ in drinking water); ${ }^{c} P<0.05$ vs LvSA ( $25 \mathrm{mg} / \mathrm{kg}$., i.p).

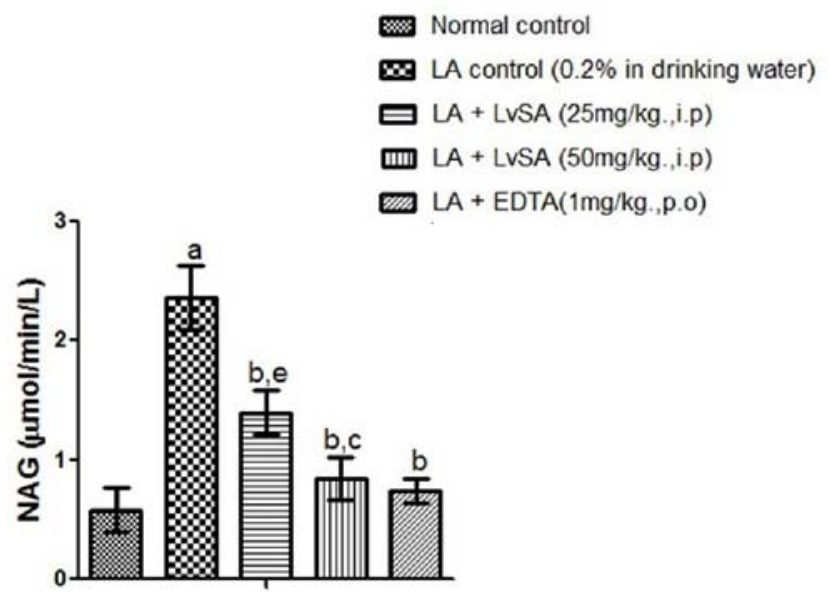

Figure 2: Effect of low viscosity sodium alginate (LvSA) on BUN (mg/dl). All values are represented as mean \pm SD. aP< 0.05 vs normal control; ${ }^{\mathrm{D}} \mathrm{P}<0.05$ vs $\mathrm{LA}$ control $(0.2 \%$ in drinking water).

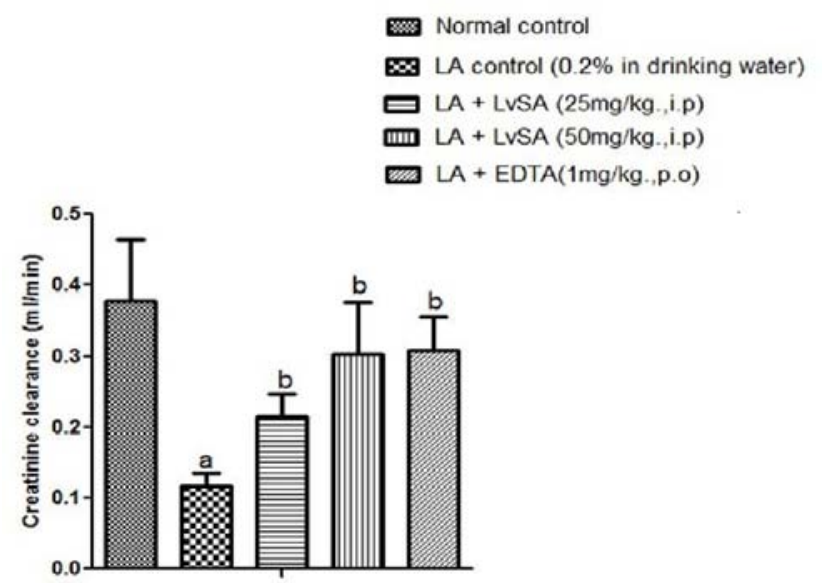

Figure 3: Effect of low viscosity sodium alginate (LvSA) on creatinine clearance $(\mathrm{ml} / \mathrm{min})$. All values are represented as mean $\pm \mathrm{SD}$. aP $<0.05$ vs normal control; ${ }^{\mathrm{b} P}<0.05$ vs LA control( $0.2 \%$ in drinking water); ${ }^{c} P<0.05$ vs $\operatorname{LvSA}(25 \mathrm{mg} / \mathrm{kg}$., i.p).

Figure 5: Effect of low viscosity sodium alginate (LvSA) on $\mathrm{NAG}(\mu \mathrm{mol} / \mathrm{min} / \mathrm{L})$. All the values are represented in mean \pm SD. aP $<0.05$ vs normal control; ${ }^{b P}<0.05$ vs LA control $(0.2 \%$ in drinking water); ${ }^{\mathrm{P}}<0.05$ vs LvSA ( $25 \mathrm{mg} / \mathrm{kg}$., i.p)

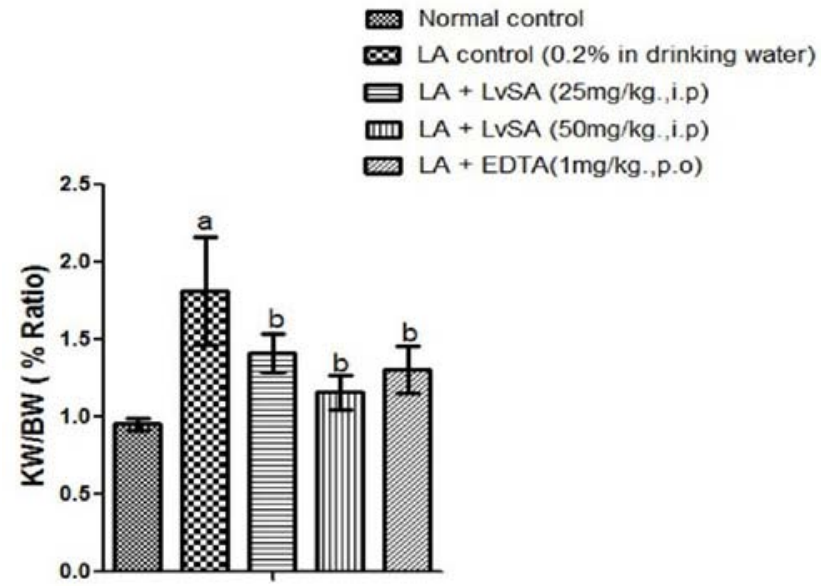

Figure 6: Effect of low viscosity sodium alginate (LvSA) on $\mathrm{KW} / \mathrm{BW} \%$. All values are represented in mean $\pm \mathrm{SD}$. ${ }^{\mathrm{P}} \mathrm{P}<0.05$ vs normal control; ${ }^{\mathrm{b}} \mathrm{P}<0.05$ vs $\mathrm{LA}$ control $(0.2 \%$ in drinking water). 


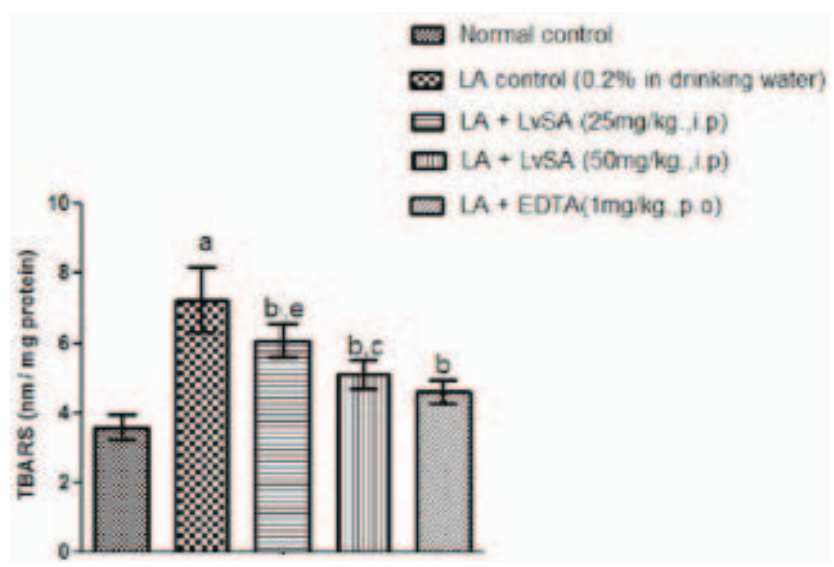

Figure 7: Effect of low viscosity sodium alginate (LvSA) on TBARS ( $\mathrm{nm} / \mathrm{mg}$ protein). All values are represented as mean $\pm \mathrm{SD}$. ${ }^{\mathrm{a} P}<0.05$ vs normal control; ${ }^{\mathrm{b}} \mathrm{P}<0.05$ vs $\mathrm{LA}$ control $(0.2 \%$ in drinking water).
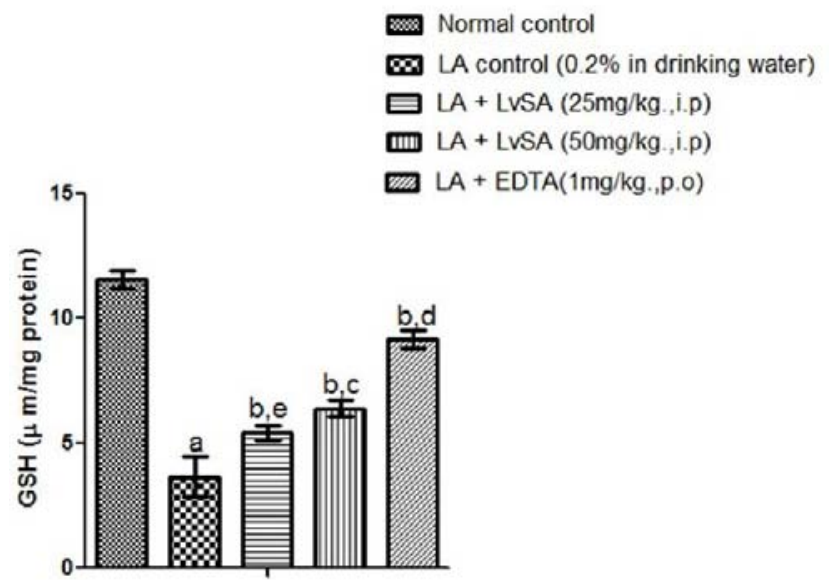

Figure 8: Effect of low viscosity sodium alginate (LvSA) on $\mathrm{GSH}(\mu \mathrm{m} / \mathrm{mg}$ protein). All values are represented in mean \pm SD. ${ }^{\text {P }}<0.05$ vs normal control; ${ }^{\mathrm{b} P}<0.05$ vs LA control $(0.2 \%$ in drinking water); ${ }^{\mathrm{P}}<0.05$ vs LvSA ( $25 \mathrm{mg} / \mathrm{kg}$., i.p).

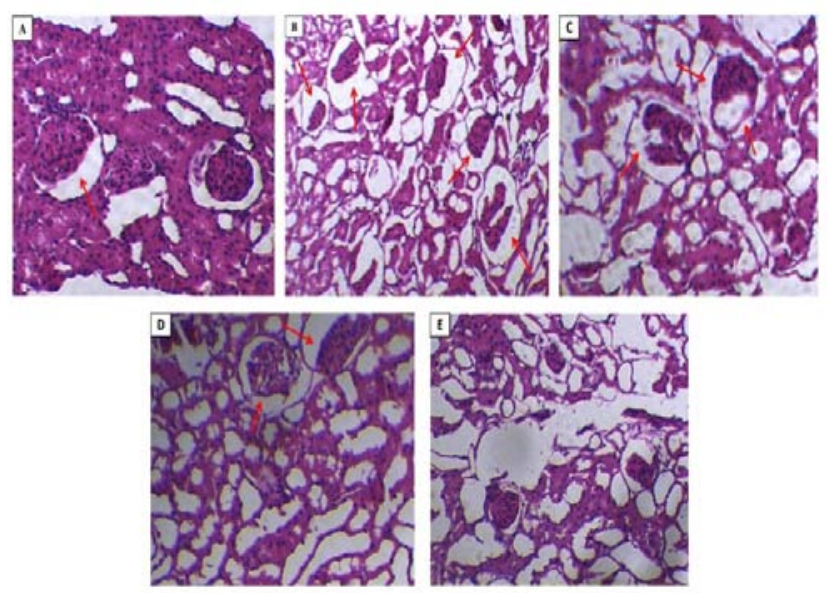

Figure 9: Histopathological view of renal sections stained with hematoxylin and eosin (10X), (A): Normal control; (B) LA; (C) LA+ LvSA(25mg/kg.,i.p); (D) LA+LvSA(50mg/kg.,i.p); (E)LA+EDTA(1 mg/kg.,p.o). Arrows indicate cortex of the kidney in fig A. Tubular epithelial necrosis and inflammation in fig B. In fig C,D,E restoration of normal cortex and reduced necrosis.

\begin{tabular}{|c|c|c|}
\hline $\begin{array}{c}\text { Table 1: Effect of various pharmacological interven- } \\
\text { tions on body weight and urine output }\end{array}$ & $\begin{array}{c}\text { Percentage } \\
\text { Parameters } \\
\text { increase in body } \\
\text { weight }\end{array}$ & $\begin{array}{c}\text { Percentage } \\
\text { increase in urine } \\
\text { output }\end{array}$ \\
\hline Normal control & $4.10 \pm 1.8$ & $4.68 \pm 2.6$ \\
\hline LA control group & $8.9 \pm 3.72^{*}$ & $4.8 \pm 6.8$ \\
\hline $\begin{array}{c}\text { LA+LvSA(25 mg/ } \\
\text { kg.,i.p) }\end{array}$ & $8.15 \pm 2.3$ & $6.7 \pm 3.7$ \\
\hline $\begin{array}{c}\text { LA+LVSA(50 mg/ } \\
\text { kg.,i.p) }\end{array}$ & $6.22 \pm 3.75^{\star}$ & $10.9 \pm 5.5^{*}$ \\
\hline $\begin{array}{c}\text { LA+ EDTA(1mg/ } \\
\text { kg.,p.o) }\end{array}$ & $2.65 \pm 1.8^{*}$ & $7.04 \pm 3$ \\
\hline
\end{tabular}

All values are represented as mean $\pm S D .{ }^{*} P<0.05$ Vs normal control; LvSA indicates low viscosity sodium alginate; LA indicates lead acetate.

(1 mg/kg p.o., 10 days) markedly protected the LA induced renal pathological changes (Figure 9).

\section{DISCUSSION}

Lead $(\mathrm{Pb})$ is an ubiquitous pollutant in the ecosystem as a result of its natural occurrence and its industrial use. Kidney is one of the organs that is generally affected because the kidney is the main route by which lead is eliminated. ${ }^{21}$ Lead is absorbed by binding to specific lead-binding proteins in the PCT of the renal tubules, these lead-protein complexes are observed as typical intracellular inclusions in acute lead nephrotoxicity. ${ }^{22}$ In order to determine the toxicity profile of lead on kidneys, different biochemical parameters and results of LA induced nephrotoxicity were compared with normal control and LvSA treated groups.

It has been reported that lead can actively make complexes with thiol or other functional groups $(-\mathrm{SH})$ and inhibits sulfahydryl-dependent enzymes. ${ }^{23}$ Present study revealed that LvSA treatment markedly reduced the increased concentration of lead in kidney tissue. This suggests that LvSA removes lead from the kidney enhancing the clearance of lead from the kidney and/ or by chelating. Sodium alginate (sodium salt of aliginic acid), which is an anionic polysaccharide extracted from the cell walls of brown algae. The mechanism involved in the metal chelation property of LvSAis not yet clearly known; so, further investigation is required.

Furthermore, consistent results are seen in body weight of LA-intoxicated rats as reported in previous studies. ${ }^{23}$ $\mathrm{KW} / \mathrm{BW} \%$ ratio was determined as an index of glomerular, mesengial expansion and renal fibrosis. A significant increase in $\mathrm{KW} / \mathrm{BW} \%$ was observed in LA administrated groups. Treatment of rats with LvSA prevented 
these observations. This suggests that LvSA has the ability to protect renal tissue from LA-induced nephrotoxicity. In the current study, we have also examined the kidney function markers, finding LA-induced elevations in serum creatinine, proteinuria, GFR, BUN, and creatinine clearance that indicate kidney dysfunction. LvSA treated rats mitigated this elevation of negative kidney function markers. Overall, present study results suggest that LvSA can help to preserve the normal function of the kidney and prevent LA-induced renal damage.

Urinary NAG appears to be the only one that is elevated in early lead nephropathy. NAG is a lysosomal enzyme present in high concentrations in the proximal tubule. ${ }^{25}$ Because of its high molecular weight, its urinary origin is unlikely to occur via glomerular sieving but rather directly from damaged kidney tissue. Dehpour et al in 1999 have shown significant increase in NAG level with LA administration. ${ }^{26}$ similar results are seen in the present study, that LA administrated groups showed a significant increase in NAG level indicating nephrotoxicity occurrence.

Lead produce its deleterious effect by attributed its ability to induce oxidative injury by generate ROS in the kidney by two separate, although related, pathways. ${ }^{21}$ The one pathway proceeds through enhancement of LPO by lead, while in the other pathway there is depletion of reserved enzymatic and nonenzymatic antioxidant molecules, whereby lead interacts with sulfhydryl groups or metal cofactors to induce a decline in antioxidant enzyme activity and in GSH. ${ }^{27}$

Increased production of epoxides, hydro peroxides, and MDA by-products of LPO are prominent marker of oxidative stress. All of which may interact with proteins, DNA, and RNA in the cell causing renal tissue damage. ${ }^{28}$ Here, LvSA has been shown to reduce LPO production and considerable effect in augment LAinduced nephrotoxicity.

The findings of the histological study support the above mentioned results, whether for the effect of LA or the preventive effects of the LvSA. The deleterious effect of LA on renal tissue, due to the oxidation actions, is a result of the overproduction of ROS, which induces cell injury and apoptosis (5). In order to have this effect, lead can easily pass into the glomerulus by endocytosis, further get reabsorbed in the proximal tubular cells. Furthermore, mitochondrial damage, glomerulosclerosis, glomerular capillary size reduction, mesangial expansion and tubular injury helps in development of LA-induced nephrotoxicity. ${ }^{29}$ To observe these changes renal tissues stained with $\mathrm{H}$ and $\mathrm{E}$. However, degeneration, desquamation, intracellular edema, glomerulus narrowing and necrosis were observed in epithelial cells of nephrons in rats of LA control group. Degenerated and desquamated epithelial cells were in lumens of tubules Figure $9 \mathrm{~B}$ is observed in some previous studies. ${ }^{30}$ significant decrease is found in renal pathological changes in LvSA administered rats Figure 9. However, further investigation should be carried out to explore the effectiveness of LvSA on LA-induced nephrotoxicity.

\section{CONCLUSION}

The current findings elucidate that LvSA can preserve renal function and structural remodeling possibly by preventing uptake and accumulation of LA in the proximal tubule. LvSA also shown beneficial effects in context to LA-induced oxidative insult. LvSA may have future therapeutic relevance in the prevention of LAinduced neprotoxicity in occupationally or environmentally exposed subjects to this toxic heavy metal. Besides, the effect nephroprotective of LvSA was not studied. Therefore, the precise mechanisms of renoprotective effect of LvSA on LA-induced nephrotoxicity await further study.

\section{ACKNOWLEDGEMENT}

The authors extend their appreciation to Mr. Parveen Garg, chairman, ISFCOP, for providing research facility.

\section{CONFLICT OF INTEREST}

No conflict of interest

\section{ABBREVIATION USED}

Glomerular filtration rate (GFR); Proximal convoluted tubules (PCT); N-acetyl $\beta$ D glucosaminidase (NAG); reduced Glutathione (GSH); kidney weight/ body weight ratio (KW/BW \%); Thiobarbituric acid reactive substances (TBARS); Blood urea nitrogen (BUN); Low viscosity sodium alginate $(\mathrm{LvSA})$.

\section{Chemical compounds studied in this article:}

Sodium alginate - PubChemCID:5102882

Lead acetate - PubChemCID:9317

EDTA - PubChemCID:6049

\section{REFERENCES}

1. Sujatha $\mathrm{K}$, Srilatha $\mathrm{CH}$. Lead acetate induced nephrotoxicity in Wistar albino rats, pathological, immunohistochemical and ultra structural studies. Int J Pharm Bio Sci2011;2:B459-69.

2. Ghosh D, Firdaus S, Mitra E, Chattopadhyay A, Pattari S, Bandyopadhyay D et al. Ameliorative effect of $S$ aqueous extract against lead acetate-induced oxidative stress in rat kidneys. Int J Pharm PharmSci 2013;5:5s46-56.

3. Loghman-Adham M. Renal effects of environmental and occupational lead exposure. Environ Health Perspect 1997;105:928. 
4. Zhang J, Cao H.Nephroprotective effect of calcium channel blockers against toxicity of lead exposure in mice. Toxicology letters. 2013;218:273-80.

5. Yang SK, Xiao L. Is lead chelation therapy effective for chronic kidney disease? A meta-analysis. Nephrology 2014;19:56-9.

6. Wang L, Feng Z.DEGseq: an $\mathrm{R}$ package for identifying differentially expressed genes from RNA-seq data. Bioinformatics. 2010;26:136-8.

7. Sears ME. Chelation: harnessing and enhancing heavy metal detoxification-a review. Scientific World J 2013. http://dx.doi.org/10.1155/2013/219840

8. Eliaz I, Weil E, Wilk B. Integrative medicine and the role of modified citrus pectin/alginates in heavy metal chelation and detoxification--five case reports. ForschKomplementmed 2007;14:358-64.

9. Bisen-Hersh EB, Farina M, Barbosa F, Rocha JB, Aschner M. Behavioral effects of developmental methylmercury drinking water exposure in rodents. J Trace Elem Med Biol 2014;28:117-24.

10. Moriya C, Shida Y. Subcutaneous Administration of Sodium Alginate Oligosaccharides Prevents Salt-Induced Hypertension in Dahl Salt-Sensitive Rats. ClinExpHypertens. 2013;35:607-13.

11. Mirshafiey A, Borzooy Z, Treatment of Experimental immune complex glomerulonephritis by sodium alginate. Vascul Pharmacol 2005;43:30-35.

12. Bauer $\mathrm{JH}$, Brooks CS.Clinical appraisal of creatinine clearance as a measurement of glomerular filtration rate. Am J Kidney Dis 1982;2:337-46.

13. Pegoraro AA, Peracha W. Evaluation of a new fluorescent dye method to measure urinary albumin in lieu of urinary total protein. Am J Kidney Dis 2000;35:739-44.

14. Kuo $\mathrm{CH}$, Hook JB. Depletion of renal glutathione content and nephrotoxicity of cephaloridine in rabbits, rats, and mice. ToxicolApplPharmacol 1982;63: 292-302.

15. Moorhead KT, Hill JV. Modelling acute renal failure using blood and breath biomarkers in rats. Comput Methods Programs Biomed 2011;101:173-82.

16. Grover JK, Vats V. Effect of feeding aqueous extract of Pterocarpus marsupium on glycogen content of tissues and the key enzymes of carbohydrate metabolism. Mol. Cell Biochem 2002;241:53-9.

17. Sinuan I, Averbukh Z. Mesengial cells initiate compensatory renal tubular hypertrophy via IL-10-induced TGF-beta secretion: effect of the immunomodulator AS101 on this process; Am J Physiol Renal Physiol 2006;291:F384-94.

\section{PICTORIAL ABSTRACT}

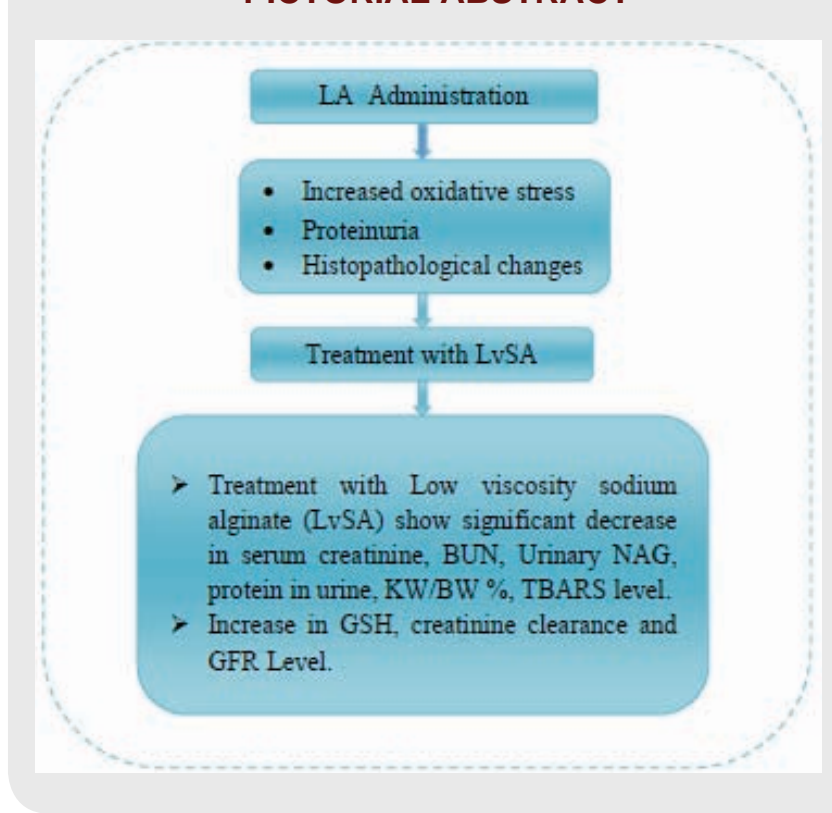

18. Kalpana C, Menon VP. Modulatory effects of curcumin on lipid peroxidation and antioxidant status during nicotine-induced toxicity. Pharmacol Rep 2013;56:581-86.

19. Tamaoka A, Miyatake F. Apolipoprotein E allele-dependent antioxidant activity in brains with Alzheimer's disease. Neurology 2000;54:2319-21.

20. Aruoma OI, Neergheen VS. Free radicals, antioxidants and diabetes: embryopathy, retinopathy, neuropathy, nephropathy and cardiovascular complications. Neuroembryology Aging 2007;4:117-37.

21. Flora G, Gupta D. Toxicity of lead: A review with recent updates. InterdiscipToxicol. 2012;5:47-58.

22. Fowler BA. Roles of lead-binding proteins in mediating lead bioavailability. Environ Health Perspect 1998;106(Suppl 6):1585.

23. Dkhil,MA, Al-Khalifa MS, Al-Quraishy $S$, Zrieq $R$, Moneim AEA. Indigoferaoblongifolia mitigates lead-acetate-induced kidney damage and apoptosis in a rat model. Drug Des Dev Ther 2016;10:1847.

24. Yavorska N. Sodium Alginate- A Potential Tool for Weight Management: Effect on Subjective Appetite, Food Intake, and Glycemic and Insulin Regulation. University of Toronto Journal of Undergraduate Life Sciences 2012;6.

25. Wolf G, Ziyadeh FN. Cellular and molecular mechanisms of proteinuria in diabetic nephropathy. Nephron Physiol 2007;106:26-31.

26. Dehpour AR, Essalat M, Ala S, Ghazi-Khansari M, Ghafourifar P. Increase by NO synthase inhibitor of lead-induced release of $\mathrm{N}$-acetyl- $\beta$ - $\mathrm{d}$ glucosaminidase from perfused rat kidney. Toxicology 1999;132:119-25.

27. Flora SJ, Mittal M, Mehta A. Heavy metal induced oxidative stress and its possible reversal by chelation therapy. Indian J Med Res. 2008;128:501-23.

28. Kehrer JP, Klotz L-O. Free radicals and related reactive species as mediators of tissue injury and disease: implications for Health. Crit Rev Toxicol. 2015;45:765-98.

29. Balakumar P, Rohilla A, Thangathirupathi A. Gentamicin-induced nephrotoxicity: do we have a promising therapeutic approach to blunt it? Pharmacol Res 2010;62,179-86.

30. Missoun F, Slimani M, Aoues A. Toxic effect of lead on kidney function in rat Wistar. Afr J Biomed Res 2010;4:21-7.

\section{SUMMARY}

- Among the toxicity profile of lead on various organs, a kidney is especially susceptible to the damaging effects of lead due to its major role in the excretion of lead from the body and is a major cause of mortality. Lead damages cellular material and produces oxidative stress by increased production of free radicals and decreased the availability of antioxidant reserves. Chelation is used as a therapy to remove toxic metals from the body, also applicable for the lead which is a heavy metal. Previous studies documented that LvSA treatment significantly reduce the urinary protein excretion and serum creatinine as well as vanquish the progression of glomerular lesions in immune complex glomerulonephritis. Therefore, we hypothesize that administration of LvSA may pre $\neg$ serve renal function, prevent histological alterations in LAinduced nephrotoxicity and it also shows beneficial effects in suppressing oxidative stress. However, the precise mechanisms of renoprotective effect of LvSA on LA-induced nephrotoxicity await further study.

Cite this article: Kaur G, Sharma S. Nephroprotective Effect of Low Viscosity Sodium Alginate on Lead Acetate -Induced Nephrotoxicity in Rats. Indian J of Pharmaceutical Education and Research. 2017;51(4S):S559-S65. 